\title{
The genera Chara and Nitella (Chlorophyta, Characeae) in the subtropical Itaipu Reservoir, Brazil
}

\author{
THAMIS MEURER ${ }^{1}$ and NORMA CATARINA BUENO ${ }^{1,2}$
}

(received: November 16, 2011; accepted: April 19, 2012)

\begin{abstract}
The genera Chara and Nitella (Chlorophyta, Characeae) in the subtropical Itaipu Reservoir, Brazil). The family Characeae, represented by two genera in Brazil, Chara and Nitella, is considered to include the closest living relatives of land plants, and its members play important ecological role in aquatic ecosystems. The present taxonomic survey of Chara and Nitella was performed in tributaries that join to form the Brazilian shore of the Itaipu Reservoir on the Paraná River. Thirteen species were recorded, illustrated, and described: C. braunii var. brasiliensis R.Bicudo, C. guairensis R.Bicudo, N. acuminata A.Braun ex Wallman, N. furcata (Roxburgh ex Bruzileus) C.Agardh, and N. subglomerata A.Braun, already cited for the reservoir, and C. hydropitys Reichenbach, C. rusbyana Howe, N. axillaris A.Braun, N. glaziovii G.Zeller, N. gracilis (Smith) C.Agardh, $N$. hyalina (DC.) C.Agardh, $N$. inversa Imahori, and $N$. microcarpa A.Braun that represent new occurrences for the Itaipu Reservoir and Paraná State. Among the species encountered, C. guairensis, N. furcata, and N. glaziovii are widely distributed, while $C$. hydropitys and C. rusbyana have more restricted distributions.
\end{abstract}

Key words - Charophyceae, macroalgae, submerged macrophyte, taxonomy

\section{INTRODUCTION}

Characeae is a unique family of algae characterized by the complexity of their morphological features, including the structure of their gametangia and their axis differentiated into nodes and internodes (PicelliVicentim et al. 2004). These algae are considered the closest living relatives of land plants (Karol et al. 2001). Characeans have important ecological role in aquatic ecosystems as their assemblages are positively correlated with water transparency, they are efficient nutrient sinks and important in nutrient cycling, and they influence zooplankton and phytoplankton biomasses (Coops 2002, Kufel \& Kufel 2002, Schwarz et al. 2002, van Donk \& van de Bund 2002, Rodrigo et al. 2007).

The genus Chara includes macroscopic algae, typically with erect corticated axes and a five-celled coronula at the apices of the female gametangia. The genus Nitella is represented by macroalgae with no cortication patterns on the axis, branches, or branchlets, and the coronula is composed of ten cells, divided into two rows of five cells each (Picelli-Vicentim et al. 2004).

The occurrence of Chara in the Itaipu Reservoir was previously reported by Thomaz et al. (1999, 2003), Meurer et al. (2008), Mormul et al. (2010), and more recently, Bueno et al. (2011), with records for Chara sp.,

\footnotetext{
1. Universidade Estadual do Oeste do Paraná - Unioeste, Campus Cascavel, Centro de Ciências Biológicas e da Saúde, Caixa Postal 711, 85814-110 Cascavel, PR. Brazil.

2._Corresponding author: ncbueno@unioeste.br
}

C. braunii var. brasiliensis, C. diaphana, C. guairensis, and $C$. kenoyeri. Information regarding Nitella in the Itaipu Reservoir is sparse, with the following species having previously been reported: N. acuminata A.Braun ex Wallman, N. furcata (Roxburgh ex Bruzileus) C. Agardh, N. furcata subsp. mucronata (A.Braun) R.D.Wood, and N. subglomerata A.Braun (Thomaz et al. 1999, 2003, Mormul et al. 2010). Considering that most of the species reported here are cited for the first time in that reservoir, this work also expands their distribution in Brazil.

The present study was carried out on the Brazilian side of the Itaipu Reservoir. Considering the important role of Characeae and the importance of acquiring more information about the species diversity of this group in Brazil, the present study surveyed the species of Chara and Nitella in the large subtropical Itaipu Reservoir in Paraná State, Brazil. The present study also provides quantitative information on relevant morphological characteristics and morphometric variations in the specimens studied, and compares them with previous records for Brazil.

\section{MATERIALS AND METHODS}

Eight tributary rivers that supply the Itaipu Reservoir (Arroio Guaçu, São Francisco Verdadeiro, São Francisco Falso, São Vicente, São João, Ocoí, Pinto, and Passo Cuê) were extensively surveyed in 2001, 2002 and 2003. We examined a total of 126 specimens of charophytes. Samples were obtained along the Brazilian shore of the Itaipu Reservoir $\left(24^{\circ} 05^{\prime}-25^{\circ} 33^{\prime} \mathrm{S}\right.$; $\left.54^{\circ} 00^{\prime}-54^{\circ} 37^{\prime} \mathrm{W}\right)$ upstream from the dam across the Paraná River. The Characeae 
specimens were collected using a small rake. In terms of nutrient concentrations, the central body of the reservoir has oligotrophic characteristics, while the tributary rivers on the left bank (Brazil) are mesotrophic to eutrophic (Bini et al. 1999).

The samples were rinsed with fresh water and analyzed using a light microscope. The specimens were subsequently preserved in Transeau solution and deposited in the UNOP herbarium (Universidade Estadual do Oeste do Paraná, Cascavel, Paraná), with duplicate samples being sent to the SP herbarium (Instituto de Botânica, São Paulo). Examinations of the biological material followed the methodologies described by Bicudo (1974); morphometric characters used in the identifications were measured in each individual examined. The classification follows Krause (1997), and the following literature was consulted for species identification and discussion: Groves \& Groves (1911), Wood \& Imahori (1964, 1965), Proctor et al. (1971), Bicudo (1974, 1977, 1979), Moore (1986), Bueno et al. (1996, 2009, 2011), Vieira Júnior et al. (2003) and Picelli-Vicentim et al. (2004).

\section{RESULTS AND DISCUSSION}

Key to the species of Chara and Nitella in the Itaipu Reservoir

1. Coronula with five cells. Presence of a cortex and/or derived structures such as stipulodes, bract-cells, or bracteoles (Genus Chara) 2

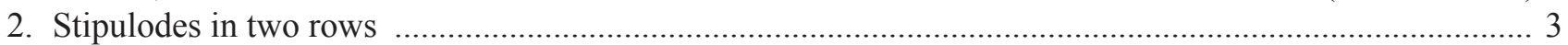

3. Plants monoecious, basal segment of branchlet fertile ...................................................... guairensis

3. Plants dioecious, basal segment of branchlet sterile C. rusbyana

2. Stipulodes in one row

4 Cortication irregular, not present on all branchlets C. hydropitys

4 Cortication absent C. hydropitys

1. Coronula with 10 cells. Absence of cortex and derived structures such as stipulodes, bract-cells, or bracteoles. (Genus Nitella) 5

5 Dimorphic, one-furcated branchlet, fertile whorls reduced in heads 6

6. One-celled dactyls N. subglomerata

6. Two-celled dactyls N. axillaris

5. Monomorphic, fertile and sterile branchlets similar 7

7. One or more furcated branchlets, one-celled dactyls N. acuminata

7. Two or more furcated branchlets, two or more celled dactyls 8

8. Accessory branchlets present, from non-furcated to bifurcated N. hyalina

8. Accessory branchlets absent 9

9. Lateral oogonia and terminal antheridia 10

9. Lateral antheridia and terminal oogonia (inverse positions) N. inversa

10. Two to four-furcated branchlets 11

11. Two to three-celled dactyls, oogonia from one to two at a node N. glaziovii

11. Two to four-celled dactyls, oogonia from one to six at a node N. furcata

10. Two to three-furcated branchlets, two to three-celled dactyls 12

12.Dactyls predominantly elongated ( $1 / 3$ of the branchlet length), oospore with granulate membrane, six to seven striae, one to two oogonia at a node N. gracilis

12.Dactyls predominantly abbreviated [94-2393(-3510) $\mu \mathrm{m}$ long], oospore with reticulate membrane, five to six striae, two to three oogonia at a node

N. microcarpa

\section{Taxonomic treatment}

Chara braunii Gmelin var. brasiliensis R.Bicudo, Rickia 8: 20, pl. 2. 1979.

Figures 1-2

Plant monoecious. Stem: $20 \mathrm{~cm}$ tall. Axes: $416-750 \mu \mathrm{m}$ diam., without incrustations. Cortex: absent. Internodes: 0.6-2.6 cm. Spine cells: absent. Stipulodes: in one tier,
291-541 $\mu \mathrm{m}$ long $\times$ 58-100 $\mu \mathrm{m}$ diam. Branchlets: 7-9(-11); 0.7-2.7 cm long, segments 5-6, ecorticated; end segment reduced, forming tiny terminal corona, $183-437 \mu \mathrm{m}$ long $\times$ 62-104 $\mu \mathrm{m}$ diam. Bract-cells: 2-4, 167-702 $\mu \mathrm{m}$ long $\times$ 62-104 $\mu \mathrm{m}$ diam. Bracteoles: 2; 604-1300 $\mu \mathrm{m}$ long $\times$ $78-130 \mu \mathrm{m}$ diam. Gametangia: at $1^{\text {st }} 2^{\text {nd }}$ lowest branchlet nodes. Oogonia: solitary, 541-1274 $\mu \mathrm{m}$ long $\times 374-702 \mu \mathrm{m}$ diam., coronula 156-286 $\mu \mathrm{m}$ long $\times 191-291 \mu \mathrm{m}$ diam., 
divergent, convolutions 10-11. Oospores: 600-754 $\mu \mathrm{m}$ long $\times 364-494 \mu \mathrm{m}$ diam., membrane finely granulate, striae 7-9, fossa 51-82 $\mu \mathrm{m}$ across. Antheridia: 312-408 $\mu \mathrm{m}$ diam., octo-scutate, triangular scute.

Representative specimens examined: BRAZIL, Paraná, Itaipu Reservoir: Passo Cuê River, 8-VIII-2002, SM Thomaz \& TA Pagioro (UNOP2918); São Francisco Falso River, 16-VIII-2002, SM Thomaz \& TA Pagioro (UNOP2037), 16-VIII-2002, SM Thomaz \& TA Pagioro (UNOP3003), 18-VIII-2002, SM Thomaz \& TA Pagioro (UNOP1850), 11-II-2003, SM Thomaz \& TA Pagioro (UNOP2995).

Geographic distribution in Brazil: in the states of Paraná (Meurer et al. 2008, Bueno et al. 2011), São Paulo (Bicudo 1979, Necchi Junior et al. 1994, 1997, 2000, Vieira Júnior et al. 2004, Picelli-Vicentim et al. 2004), and Rio Grande do Sul (Bueno et al. 2011).

Comments: Chara braunii var. brasiliensis resembles C. socotrensis Nordstedt emend. R.D.Wood due to the presence of gametangia on the basal segment of the branchlet, they differ because $C$. braunii has a crown of bract-cells on each branchlet apex, which are absent in C. socotrensis.

The var. brasiliensis was described by Bicudo (1979) and the sample specimens studied here are in accordance with the description presented in that study. Casanova (2005) notes that similar specimens sampled on different continents should eventually be considered different species, varieties, or taxonomical forms, as was pointed out by Proctor (1971), for this reason we decided to maintain the variety designation of Bicudo.

Chara guairensis R.Bicudo, Rickia 6: 145, pl. 4, fig. 1-11. 1974.

Figures 3-6

Plant monoecious. Stem: up to $40 \mathrm{~cm}$ tall. Axes: 460-1083 $\mu \mathrm{m}$ diam. Internodes: 0.7-7.3 cm long. Cortex: triplostichous. Spine cells: solitary, 31-396 $\mu \mathrm{m}$ long $\times$ 41-93 $\mu \mathrm{m}$ diam. Stipulodes: in two developed rows, upper row 423-1352 $\mu \mathrm{m}$ long $\times 75-150 \mu \mathrm{m}$ diam., lower row (208-)333-1400 $\mu \mathrm{m}$ long $\times 74-133 \mu \mathrm{m}$ diam. Branchlets: 9-13(-15); 0.9-5.5 cm long $\times 360-540 \mu \mathrm{m}$ diam., basal segment ecorticated, 540-1290 $\mu \mathrm{m}$ long $\times$ 300-479 $\mu \mathrm{m}$ diam., intermediate segments 6-12, corticated, apical segment ecorticated, with bract-cells. Bract-cells: 5-6, verticilate, $155-1978 \mu \mathrm{m}$ long $\times 62-$ $180 \mu \mathrm{m}$ diam. Bracteoles: 4; 580-1560 $\mu \mathrm{m}$ long $\times 80$ $250 \mu \mathrm{m}$ diam. Gametangia: sejoined from $1^{\text {st }}$ to $6^{\text {th }}$ nodes. Oogonia: solitary, 725-1040 $\mu \mathrm{m}$ long $\times 396-568 \mu \mathrm{m}$ diam., coronula 114-208 $\mu \mathrm{m}$ long $\times 192-291 \mu \mathrm{m}$ diam., divergent, convolutions 11-12. Oospore: 441-666 $\mu \mathrm{m}$ long $\times 312-442 \mu \mathrm{m}$ diam., membrane granulate, striae 9-11, fossa 40-78 $\mu \mathrm{m}$ across. Antheridia: 237-598 $\mu \mathrm{m}$ diam., octo-scutate, triangular scute.

Representative specimens examined: BRAZIL, ParAnÁ, Itaipu Reservoir: Passo Cuê River, 08-VIII-2002, SM Thomaz \& TA Pagioro (UNOP2073); Ocoí River, 14-VIII-2002, SM Thomaz \& TA Pagioro (UNOP2080); São João River, 12-VIII-2002, SM Thomaz \& TA Pagioro (UNOP2108), 12-VIII-2003, SM Thomaz \& TA Pagioro (UNOP1843), 20-II-2003, SM Thomaz \& TA Pagioro (UNOP2180); São Vicente River, 13-VIII-2002, SM Thomaz \& TA Pagioro (UNOP2922), 13-II-2003, SM Thomaz \& TA Pagioro (UNOP1796, SP371087); Pinto River, 25-II-2003, SM Thomaz \& TA Pagioro (UNOP1664, SP371093), 27-II-2003, SM Thomaz \& TA Pagioro (UNOP2149), 27-II-2003, SM Thomaz \& TA Pagioro (UNOP2152).

Geographic distribution in Brazil: in the states of Mato Grosso (Bueno et al. 2009), Mato Grosso do Sul (Bueno et al. 1996, 2009), Paraná (Bicudo 1974, Bueno et al. 2011), São Paulo (Vieira Júnior \& Necchi Júnior 2002, Vieira Júnior et al. 2003, Picelli-Vicentim et al. 2004), and Rio Grande do Sul (Bueno et al. 2011).

Comments: Chara guairensis is characterized by having long bract-cells, sejoined gametangia, and a fertile basal segment. The species was recorded in the Itaipu Reservoir at different sampling stations. Our results extend the distribution of this species in the reservoir and contribute to a more accurate knowledge of Chara communities in the Itaipu River.

Chara hydropitys Reichenbach in Möessler's Handbuch der Gewächskunde 3: 1669. 1834.

Figures 7-9

Plant monoecious. Stem: $15 \mathrm{~cm}$ tall. Axes: 590$728 \mu \mathrm{m}$ diam. Internodes: o correto é: $0.3-1$ times the branchlet lenght. Cortex: triplostichous, isostichous. Spine cells: small to obscure, $31-33 \mu \mathrm{m}$ long $\times 42 \mu \mathrm{m}$ diam. Stipulodes: obscure, in one tier, 433-649 $\mu \mathrm{m}$ long $\times$ 62-82 $\mu \mathrm{m}$ diam. Branchlets: 9-12, intermediate segments (5-)6-7, 0-2-corticated; basal segment ecorticated; end segment ecorticated with bract-cells. Bract-cells: 4-6; $(158-) 1706 \mu \mathrm{m}$ long $\times 50-156 \mu \mathrm{m}$ diam. Bracteoles: 1040-1612 $\mu \mathrm{m}$ long $\times 50-156 \mu \mathrm{m}$ diam. Gametangia: conjoined from $1^{\text {st }}$ to $4^{\text {th }}$ nodes. Oogonia: $716-837 \mu \mathrm{m}$ long $\times$ 450-566 $\mu \mathrm{m}$ diam., coronula 100-108 $\mu \mathrm{m}$ long $\times$ 192-225 $\mu \mathrm{m}$ diam., divergent, convolutions 11-12. Oospores: $700 \mu \mathrm{m}$ long $\times 300 \mu \mathrm{m}$ diam., membrane smooth, striae 10-11. Antheridia: 312-338 $\mu \mathrm{m}$ diam., octo-scutate, scute triangular. 


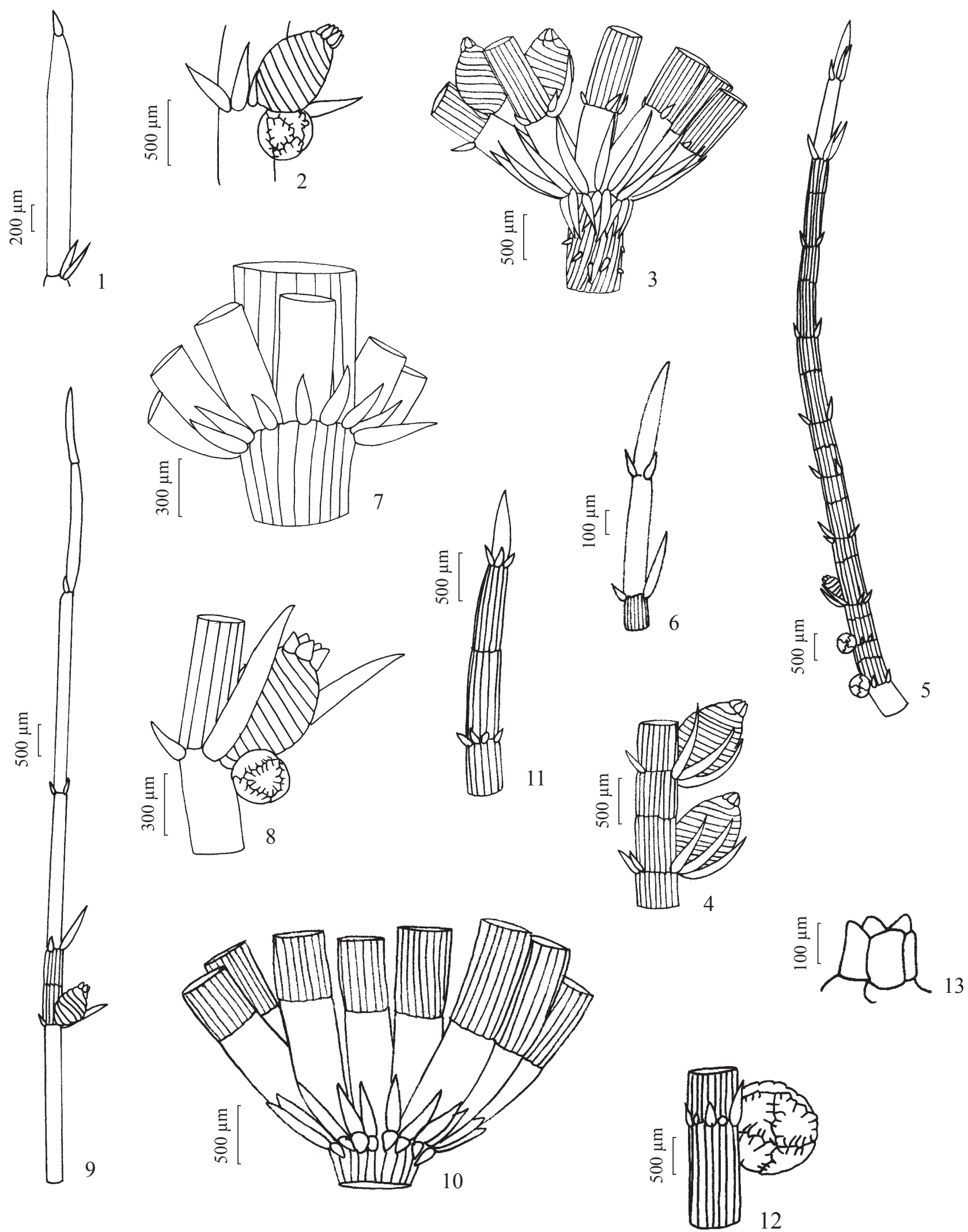

Figures 1-13. The genus Chara from the Itaipu Reservoir. 1-2. Chara braunii var. brasiliensis. 1. Branchlet apex. 2. Fertile node with conjoined gametangia. 3-6. C. guairensis. 3. Diplostephanous stipulodes. 4. Sejoined gametangia. 5. Fertile branchlet. 6. Branchlet apex. 7-9. C. hydropitys. 7. Haplostefanous stipulodes. 8. Conjoined gametangia. 9. Branchlet with one-corticated segment. 10-13. C. rusbyana. 10. Diplostephanous stipulodes. 11. Branchlet apex. 12. Antheridium. 13. Coronula. 
Representative specimens examined: BRAZIL, PARANÁ, Itaipu Reservoir: São João River, 14-II-2002, SM Thomaz \& TA Pagioro (UNOP1745).

Geographic distribution in Brazil: as C. fibrosa var. hydropitys: Bahia (Bicudo 1974), Maranhão (Bicudo 1974), Mato Grosso (Bueno et al. 2009), Mato Grosso do Sul (Bicudo 1974, Bueno et al. 1996, 2009), Rio Grande do Sul (Prado 2003, Bueno et al. 2011).

Comments: This is the first report of Chara hydropitys for Paraná State. An extensive discussion concerning the species can be found in the recent publication of Bueno et al. (2011). The specimens analyzed in this study had nine to 12 branchlets per whorl, with a maximum of two corticated segments, in accordance with the original description of the species. All other diagnostic characteristics for $C$. hydropitys fall within the morphometric limits presented for other specimens described in previous studies in Brazil.

Chara rusbyana Howe, Field Museum of Natural History 4(6): 160. 1929.

Figures 10-13

Plants dioecious. Axes: $650 \mu \mathrm{m}$ diam. Internodes: 1-1.3 cm long. Cortex: triplostichous, isostichous. Spine cells: $134 \mu \mathrm{m}$ long $\times 82 \mu \mathrm{m}$ diam. Stipulodes: in two developed rows, upper row 728-754 $\mu \mathrm{m}$ long $\times$ up to $78 \mu \mathrm{m}$ diam., lower row 312-433 $\mu \mathrm{m}$ long $\times 130-390 \mu \mathrm{m}$ diam. Branchlets: 9-14; basal segment ecorticated, intermediate segments 6-12, corticated; apical segment 1-2, ecorticated, with bract-cells. Bract-cells: 5-8;257 $\mu \mathrm{m}$ long $\times 82 \mu \mathrm{m}$ diam. Bracteoles: $2 ; 910 \mu \mathrm{m}$ long $\times 78 \mu \mathrm{m}$ diam. Gametangia: sejoined at $2^{\text {nd }}-5^{\text {th }}$ branchlet nodes. Oogonia:solitary; $1014 \mu \mathrm{mlong} \times 520 \mu$ mdiam.Coronula: convergent or divergent, $130 \mu \mathrm{m}$ long $\times 208 \mu \mathrm{m}$ diam., convolutions 12. Oospores: $624 \mu \mathrm{m}$ long $\times 442 \mu \mathrm{m}$ diam., membrane homogeneous, striae 10. Antheridia: not seen.

Representative specimens examined: BRAZIL, ParanÁ, Itaipu Reservoir: Ocoí River, 14-VIII-2002, SM Thomaz \& TA Pagioro (UNOP1599).

Geographic distribution in Brazil: in the states of Mato Grosso (Braun \& Nordstedt 1883, Howe 1929, Bicudo 1974), Mato Grosso do Sul (Bueno et al. 1996, 2009), Minas Gerais, Pernambuco, Piauí, Santa Catarina (Braun \& Nordstedt 1883, Bicudo 1974, Bueno et al. 2011), São Paulo (Braun \& Nordstedt 1883, Bicudo 1974, Picelli-Vicentim et al. 2004), and Rio Grande do Sul (Bueno et al. 2011).

Comments: According to Proctor (1971), this species is restricted to South America. It resembles
Chara kenoyeri Howe, from which it differs in having smaller bract cells and fewer segments in the branchlets. This is the first record of this species for Paraná State.

Nitella acuminata A.Braun ex Wallman, Försök till en systematisk uppställning af växfamiljen Characeae 35. 1853.

Figure 14

Plants monoecious. Stem: $15 \mathrm{~cm}$ tall. Axes: 416$676 \mu \mathrm{m}$ diam. Internodes: $1-3.2 \mathrm{~cm}$ long. Branchlets: monomorphic, 6-8, 1-furcated, 1-2 cm long. Primary rays: 6-8, 6-7 mm long. Dactyls: 2-5, 1-celled, 2-7.8 mm long $\times 112-312 \mu \mathrm{m}$ diam. Fertile heads: absent. Gametangia: conjoined, sessile, occasionally with a short stalk. Oogonia: one or two at a node, $330-453 \mu \mathrm{m}$ long $\times 288$ $340 \mu \mathrm{m}$ diam., convolutions 9-10, coronula 31-41 $\mu \mathrm{m}$ long $\times$ 41-51 $\mu \mathrm{m}$ diam. Oospore: $247-319 \mu \mathrm{m}$ long $\times 196-$ $227 \mu \mathrm{m}$ diam., striae 7 , fossa $42-55 \mu \mathrm{m}$ across, membrane finely granulate. Antheridia: (154-)185-319 $\mu \mathrm{m}$ diam., octo-scutate, triangular scute.

Representative specimens examined: BRAZIL, PARANÁ, Itaipu Reservoir: São João River, 22-II-2002, SM Thomaz \& TA Pagioro (UNOP1767); Ocoí River, 19-II-2003, SM Thomaz \& TA Pagioro (UNOP2117); 11-I-2001, SM Thomaz \& TA Pagioro (UNOP1643); Pinto River, 27-I-2002 (UNOP1305).

Geographic distribution in Brazil: in the states of Mato Grosso do Sul (Bueno \& Bicudo 1997), Paraná (Thomaz et al. 2003), Rio de Janeiro (Bicudo 1969, Bicudo \& Yamaoka 1978), São Paulo (Bicudo 1969, Bicudo \& Yamaoka 1978, Picelli-Vicentim \& Bicudo 1993, Picelli-Vicentim et al. 2004), and Rio Grande do Sul (Prado 2003).

Comments: The specimens examined were in accordance with the diagnostic characteristics for $N$. acuminata, including one-celled dactyls, monomorphic whorls, and one-furcated branchlets (Bicudo 1969, Bicudo \& Yamaoka 1978, Picelli-Vicentim et al. 2004). The morphometric variations of the observed structures were in accordance with previously published limits for the country. Sample UNOP1767 had the largest oogonia (up to $453 \mu \mathrm{m}$ long) and antheridia (up to $319 \mu \mathrm{m}$ diameter), while UNOP1305 had the largest oospores (up to $319 \mu \mathrm{m}$ long).

Nitella axillaris A.Braun, Monatsbericht der deutschen Akademie der Wissenschaften zu Berlin 356. 1858. Figures 15-16

Plants monoecious. Stem: $35-40 \mathrm{~cm}$ tall; without incrustations. Axes: 468-1092 $\mu \mathrm{m}$ diam. Internodes: 
1-6.7 cm long. Branchlets: dimorphic, 1-furcated, fertile reduced. Sterile branchlets: 6-9, appearing simple, 1-furcated, 0.5-3 cm long $\times 260-1000 \mu \mathrm{m}$ diam., primary rays 6-9, as long as branchlets, 0.4-2.7 cm long, dactyls 3-5, 2-celled, forming a mucro, 312-962 $\mu \mathrm{m}$ long $\times 78$ $260 \mu \mathrm{m}$ diam. Fertile branchlets: 7-8; 1-furcated, 1290$1648 \mu \mathrm{m}$ long, forming heads, dactyls 3-5, 2-celled, 286-650 $\mu \mathrm{m}$ long $\times 62-156 \mu \mathrm{m}$ diam. Heads: axillary, 1-3 per whorl, occasionally with a stalk, 1-3 mm diam. Gametangia: conjoined. Oogonia: 1-3 at a node, 288$515 \mu \mathrm{m}$ long $\times 247-381 \mu \mathrm{m}$ diam., convolutions 8-9, coronula 31-51 $\mu \mathrm{m}$ long $\times 50-72 \mu \mathrm{m}$ diam. Oospore: 247-330 $\mu \mathrm{m}$ long $\times 237-288 \mu \mathrm{m}$ diam., membrane reticulate, striae 6-7, fossa 40-70 $\mu \mathrm{m}$ across. Antheridia: 154-598 $\mu \mathrm{m}$ diam., octo-scutate, triangular scute.

Representative specimens examined: BRAZIL, PARANÁ, Itaipu Reservoir: São Francisco Falso River, 25-IV-2002, SM Thomaz \& TA Pagioro (UNOP1357); São Vicente River, 13-VIII-2002, SM Thomaz \& TA Pagioro (UNOP2921); 31-I-2002, SM Thomaz \& TA Pagioro (UNOP1342); São João River, 20-II-2003, SM Thomaz \& TA Pagioro (UNOP1750); 22-II-2003, SM Thomaz \& TA Pagioro (UNOP2895); Ocoí River, 17-VIII-2002, SM Thomaz \& TA Pagioro (UNOP1641); 23-I-2002, SM Thomaz \& TA Pagioro (UNOP1304); 14-VIII-2002, SM Thomaz \& TA Pagioro (UNOP1618); 14-VIII-2002, SM Thomaz \& TA Pagioro (UNOP2086); 19-III-2003, SM Thomaz \& TA Pagioro (UNOP1621); 27-I-2002, SM Thomaz \& TA Pagioro (UNOP1309); 14-VIII-2002, SM Thomaz \& TA Pagioro (UNOP1632).

Geographic distribution in Brazil: [as Nitella translucens (Persoon) C.Agardh emend. R.D.Wood subsp. translucens var. axillaris (A.Braun) R.D.Wood f. axillaris] in the states of Mato Grosso do Sul (Bueno \& Bicudo 1997), Pernambuco (Wood \& Imahori 1965), São Paulo (Picelli-Vicentim \& Bicudo 1993, Picelli-Vicentim et al. 2004), and Rio Grande do Sul (Prado 2003).

Comments: This is the first record of Nitella axillaris for the Itaipu Reservoir, and the first record of the species for Paraná State. The material analyzed here agreed with the descriptions given by Wood \& Imahori $(1964,1965)$, Picelli-Vicentim \& Bicudo (1993), and Bueno \& Bicudo (1997). The upper morphometric limits of antheridia increased from $250 \mu \mathrm{m}$ diameter (Prado 2003) to $598 \mu \mathrm{m}$ (UNOP1357). Specimens from the Ocoí River had the largest oogonia and oospores (UNOP2086) as well as the smallest antheridia (UNOP2086) and oospores (UNOP1632). The largest antheridia were observed in material collected in the São Francisco Falso River (UNOP1357).
Nitella axillaris was treated as a variety of Nitella translucens by Wood \& Imahori (1965). Moore (1986) reported that the oospores of $N$. translucens were longer, ovoid, and that its membrane ornamentation was finely reticulated, with five or six striae. Sakayama et al. (2002) reported that $N$. axillaris oospores were ovoid and had a membrane with a strongly reticulate ornamentation pattern, with six or seven striae. Molecular phylogenetic analyses have shown that $N$. axillaris and $N$. translucens are different species (Sakayama 2008).

Nitella furcata (Roxburgh ex Bruzileus) C.Agardh. Systema Algarum, p.124. 1824.

Figures 17-21

Plants monoecious. Stem: 21(-40) cm tall; without incrustations. Axes: 312-910 $\mu \mathrm{m}$ diam. Internodes: 0.6$5.7 \mathrm{~cm}$ long. Branchlets: monomorphic, 5-8, 2-3(-4)furcated, $0.8-3.8 \mathrm{~cm}$ long. Primary rays: $5-8,0.3-1.7 \mathrm{~cm}$ long. Secondary rays: 4-6. Tertiary rays: $3-5$. Quaternary rays: 2-4. Dactyls: 2-3(-4), abbreviated, 2-3-celled, 113$3380 \mu \mathrm{m}$ long $\times 52-208 \mu \mathrm{m}$ diam., end cells conical, acuminate or acute. Heads: not formed. Gametangia: conjoined or sejoined, occasionally with a short stalk. Oogonia: 1-5(-6) at a node, 309-624 $\mu \mathrm{m}$ long $\times 237-$ $443 \mu \mathrm{m}$ diam., convolutions 6-9, coronula convergent, (31-)41-77 $\mu \mathrm{m}$ long $\times 50-93 \mu \mathrm{m}$ diam. Oospore: 227 $319 \mu \mathrm{m}$ long $\times 175-330 \mu \mathrm{m}$ diam., striae 5-7, fossa 40$82 \mu \mathrm{m}$ across, membrane reticulate. Antheridia (144-) 175-364 $\mu \mathrm{m}$ diam., octo-scutate, triangular scute.

Representative specimens examined: BRAZIL, ParanÁ, Itaipu Reservoir: São Francisco Falso River, 24-XII-2003, SM Thomaz \& TA Pagioro (UNOP1728); 25-IV-2002, SM Thomaz \& TA Pagioro (UNOP1351); 10-X-2002, SM Thomaz \& TA Pagioro (UNOP1773); 12-XI-2002, SM Thomaz \& TA Pagioro (UNOP1692); 24-II-2003, SM Thomaz \& TA Pagioro (UNOP1691); 16-VIII-2002, SM Thomaz \& TA Pagioro (UNOP1702); 26-II-2002, SM Thomaz \& TA Pagioro (UNOP2902); 16-II-2002, SM Thomaz \& TA Pagioro (UNOP1708); 26-VI-2001, SM Thomaz \& TA Pagioro (UNOP1711); 11-II-2003, SM Thomaz \& TA Pagioro (UNOP2129); 11-II-2003, SM Thomaz \& TA Pagioro (UNOP1815); 11-II-2003, SM Thomaz \& TA Pagioro (UNOP1718); 11-II-2003, SM Thomaz \& TA Pagioro (UNOP1719); 11-II-2003, SM Thomaz \& TA Pagioro (UNOP1724); 11-II-2003, SM Thomaz \& TA Pagioro (UNOP1723); São Vicente River, 13-VIII-2002, SM Thomaz \& TA Pagioro (UNOP1777); 13-VIII-2002, SM Thomaz \& TA Pagioro (UNOP3005); 13-II-2003, SM Thomaz \& TA Pagioro (UNOP1788); 13-II-2003, SM Thomaz \& TA Pagioro 

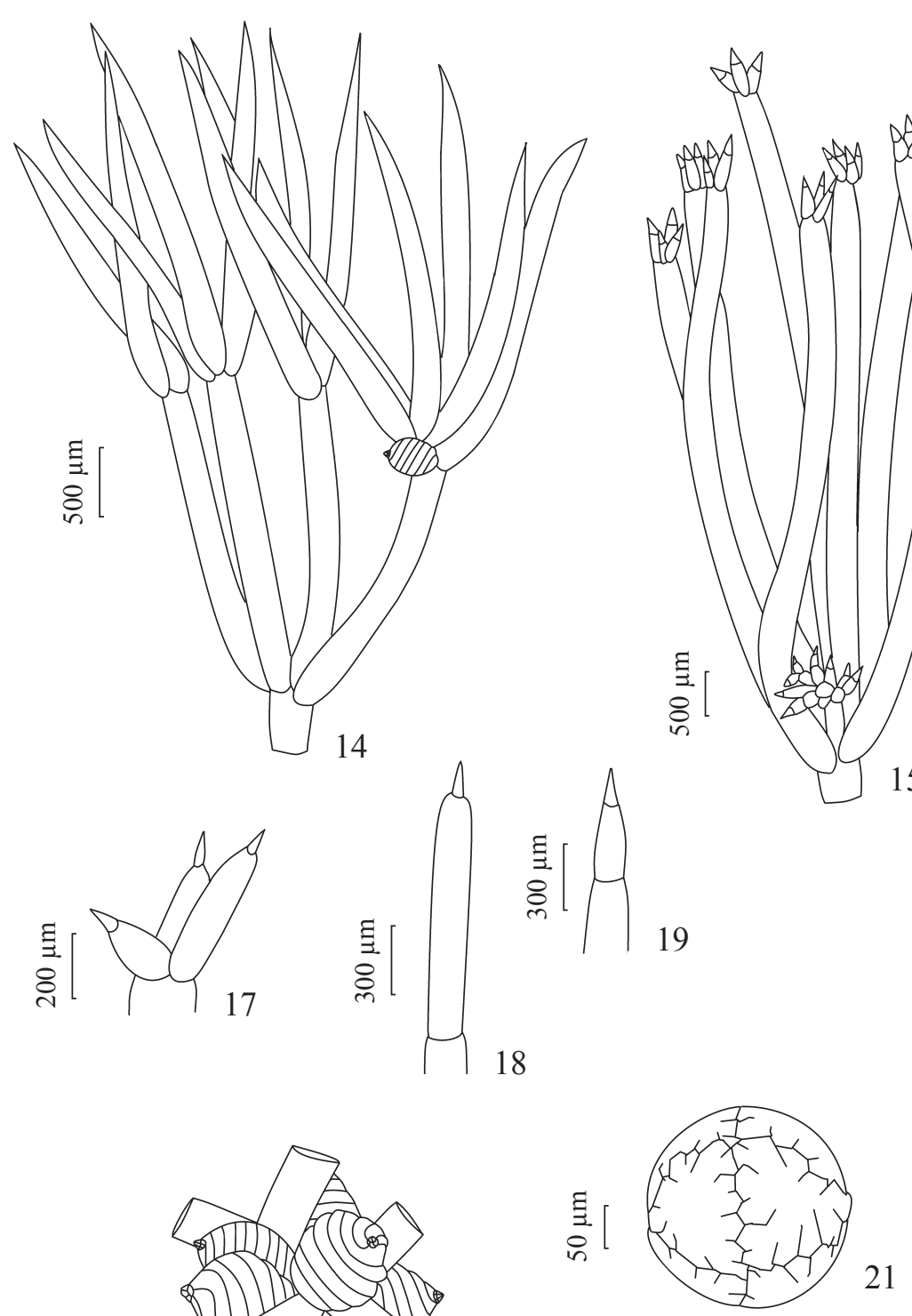

19

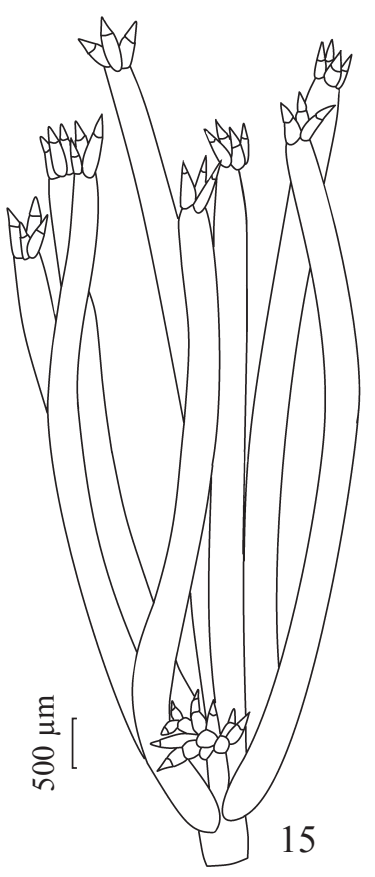

15
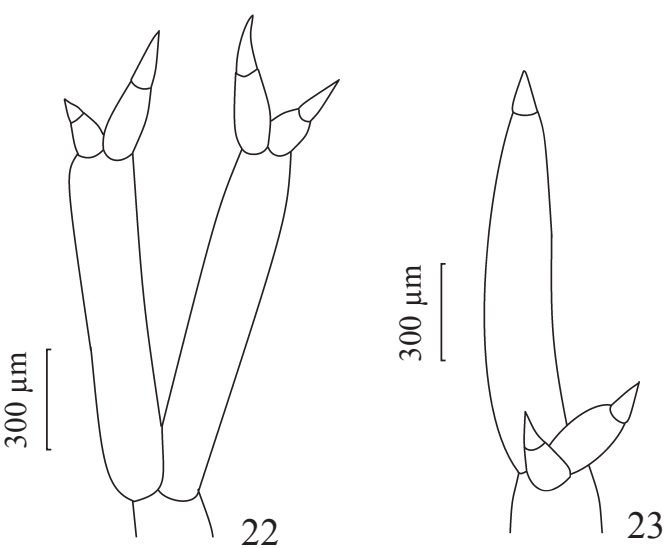
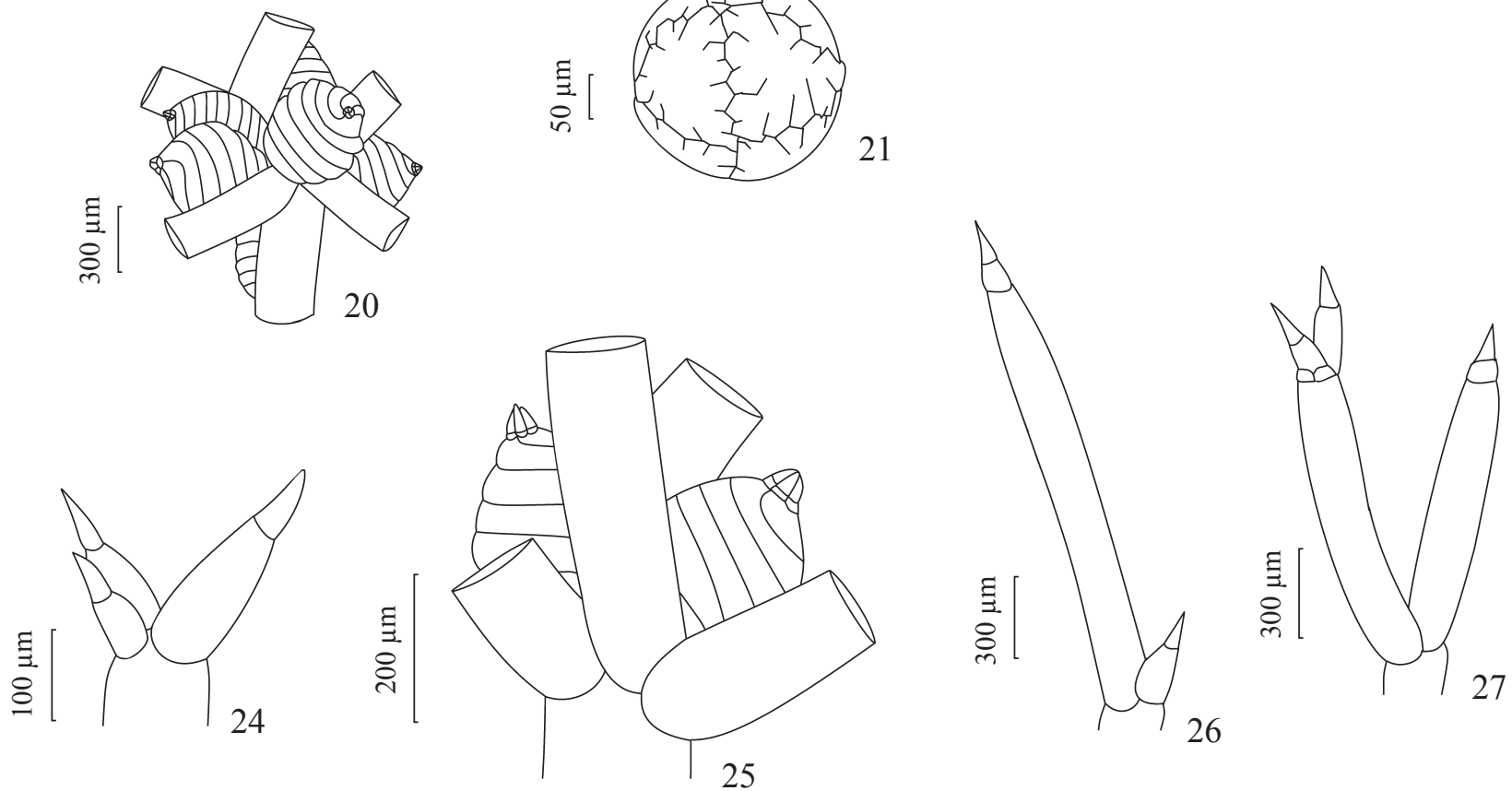

Figures 14-27. The genus Nitella from the Itaipu Reservoir. 14. Nitella acuminata, fertile branchlet. 15-16. N. axillaris. 15. Branchlet. 16. Fertile head. 17-21. N. furcata. 17-19. Dactyls. 20. Node with six oogonia. 21. Antheridium. 22-27. N. glaziovii. 22-24. Two-celled dactyls. 25. Oogonium. 26-27. Two and three-celled dactyls. 
(UNOP1790); 12-VIII-2002, SM Thomaz \& TA Pagioro (UNOP1791); 13-VIII-2002, SM Thomaz \& TA Pagioro (UNOP1799); 13-VIII-2002, SM Thomaz \& TA Pagioro (UNOP1807); São João River, 12-VIII-2002, SM Thomaz \& TA Pagioro (UNOP1733); 12-VIII-2002, SM Thomaz \& TA Pagioro (UNOP1836); 20-II-2003, SM Thomaz \& TA Pagioro (UNOP2187); 12-VIII-2002, SM Thomaz \& TA Pagioro (UNOP2105); 12-VIII-2002, SM Thomaz \& TA Pagioro (UNOP2108); 12-VIII-2002, SM Thomaz \& TA Pagioro (UNOP1749); Ocoí River, 11-I-2001, SM Thomaz \& TA Pagioro (UNOP1642); 14-VIII-2002, SM Thomaz \& TA Pagioro (UNOP1613); 14-VIII-2002, SM Thomaz \& TA Pagioro (UNOP1627); 14-VIII-2002, SM Thomaz \& TA Pagioro (UNOP1603); 14-VIII-2002, S. M. Thomaz \& T.A. Pagioro (UNOP1620); 14-VIII-2002, SM Thomaz \& TA Pagioro (UNOP1623); 14-VIII-2002, SM Thomaz \& TA Pagioro (UNOP1625); 14-VIII-2002, SM Thomaz \& TA Pagioro (UNOP2080); 14-VIII-2002, SM Thomaz \& TA Pagioro (UNOP2079); 14-VIII-2002, SM Thomaz \& TA Pagioro (UNOP1630); 27-VIII-2001, SM Thomaz \& TA Pagioro (UNOP1640); Pinto River, 09-VIII-2002, SM Thomaz \& TA Pagioro (UNOP1661); 09-VIII-2002, SM Thomaz \& TA Pagioro (UNOP1670); 27-II-2003, SM Thomaz \& TA Pagioro (UNOP1672); 09-VIII-2002, SM Thomaz \& TA Pagioro (UNOP1677); 09-VIII-2002, SM Thomaz \& TA Pagioro (UNOP2071); 09-VIII-2002, SM Thomaz \& TA Pagioro (UNOP1673); 27-II-2003, SM Thomaz \& TA Pagioro (UNOP2152); 27-II-2003, SM Thomaz \& TA Pagioro (UNOP1676); 09-VIII-2002, SM Thomaz \& TA Pagioro (UNOP1679); Passo Cuê River, 26-II-2002, SM Thomaz \& TA Pagioro (UNOP1652); 26-II-2003, SM Thomaz \& TA Pagioro (UNOP1656); 26-II-2003, SM Thomaz \& TA Pagioro (UNOP1659).

Geographic distribution in Brazil: Paraná - Itaipu Reservoir (Thomaz et al. 1999), Rio Grande do Sul (Prado 2003).

Comments: Nitella furcata is a monoecious species, with homoclemous branchlets, absence of gametangia on basal furcation, with mucronate and predominantly abbreviated dactyls that form a cuspidate crown; fertile heads are rarely formed (Zaneveld 1940, Horn af Rantzien 1949, Wood \& Imahori 1965). The samples identified as Nitella furcata had a reticulate ornamentation pattern of oospores, as reported by Mandal et al. (1995) and Sakayama et al. (2002).

The highest morphometric values for oogonia and oospores were recorded for UNOP1670 and UNOP1677 respectively. Sample UNOP1672, from the Pinto River, had the smallest antheridia. Compared to the morphometric values observed by Prado (2003), the present study expanded the upper morphometric limit of the dactyls from $1260 \mu \mathrm{m}$ to $3380 \mu \mathrm{m}$ long, the antheridia diameter from $310 \mu \mathrm{m}$ to $364 \mu \mathrm{m}$, and the lower limit of the coronula from $52 \mu \mathrm{m}$ to $31 \mu \mathrm{m}$ long.

Nitella glaziovii G.Zeller, In Warming (ed.), Symbolae ad Floram Brasiliae Centralis Cognoscendam. Vidensk. Meddel. Naturhist. Foren. Kjøbenhavn 1876, $12427-$ 435. 1876.

Figures 22-27

Plants monoecious, without incrustations. Axes: 360$780 \mu \mathrm{m}$ diam. Internodes: $1.4-4.5 \mathrm{~cm}$ long. Branchlets: monomorphic, 2-3-4 furcated, 1-2.7 cm long. Primary rays: $7-8,0.2-1.4 \mathrm{~mm}$ long. Secondary rays: $4-5$, one of which is occasionally central and reduced. Tertiary rays: 3-4. Quaternary rays: 2-3. Dactyls: 2-3, 2-3-celled, 130-2,548 $\mu \mathrm{m}$ long $\times 52-156 \mu \mathrm{m}$ diam., predominantly abbreviated, conical end cell, acute apex. Heads: not formed. Gametangia: conjoined at all furcations. Oogonia: $1-2$ at a node, (268-)309-566 $\mu \mathrm{m}$ long $\times 299$ $468 \mu \mathrm{m}$ diam., convolutions 6-8, coronula 45-75 $\mu \mathrm{m}$ long $\times 60-80 \mu \mathrm{m}$ diam. Oospore 225-309 $\mu \mathrm{m}$ long $\times$ 185-257 $\mu \mathrm{m}$ diam., striae 5-6, fossa $45-63 \mu \mathrm{m}$ long, membrane reticulate. Antheridia: $165-312 \mu \mathrm{m}$ diam., octo-scutate, triangular scute.

Representative specimens examined: BRAZIL, ParanÁ, Itaipu Reservoir: Arroio Guaçu river, 10-II2003, SM Thomaz \& TA Pagioro (UNOP1584); São Francisco Falso River, 24-II-2003, SM Thomaz \& TA Pagioro (UNOP1694); 16-VIII-2002, SM Thomaz \& TA Pagioro (UNOP1700); 12-XI-2002, SM Thomaz \& TA Pagioro (UNOP1690); São Vicente River, 13-II-2003, SM Thomaz \& TA Pagioro (UNOP2171, UNOP1808); São João River, 20-II-2003, SM Thomaz \& TA Pagioro (UNOP1817); Ocoí River, 25-II-2003, SM Thomaz \& TA Pagioro (UNOP1633); Pinto River, 27-II-2003, SM Thomaz \& TA Pagioro (UNOP2149); 25-II-2003, SM Thomaz \& TA Pagioro (UNOP1662); Passo Cuê River, 08-VIII-2002, SM Thomaz \& TA Pagioro (UNOP1649); 08-VIII-2002, SM Thomaz \& TA Pagioro (UNOP1651); SM Thomaz \& TA Pagioro (UNOP2075).

Geographic distribution in Brazil: Rio de Janeiro (Wood \& Imahori 1964, 1965 [= Nitella furcata (Roxb. ex Bruz.) Ag. emend. R.D.Wood var. sieberi (A.Braun) R.D.Wood f. glaziovii]).

Comments: This is the first record of this species in the Itaipu Reservoir and also the first record of this species for Paraná State. Wood \& Imahori $(1964,1965)$ mentioned its occurrence in Rio de Janeiro state. The citation of $N$. glaziovii by Bicudo (1969) was later 
corrected by the author, and was then identified as $N$. furcata subsp. furcata var. sieberi f. japonica.

$N$. glaziovii has one or two oogonia at each furcation, branchlets have up to four furcations, and the oospore ornamentation pattern is similar to the reticulate pattern described by Wood \& Imahori (1965) and Sakayama (2008). N. glaziovii resembles $N$. gracilens in terms of their numbers of gametangia and the presence of predominantly abbreviated dactyls. However, $N$. gracilens, which has only been recorded in Japan, has up to three furcations on each branchlet and the ornamentation of oospore membrane is finely granulated - characteristics not observed in the samples from the Itaipu Reservoir.

Nitella gracilis (Smith) C.Agardh, Systema Algarum, p.125. 1824.

Figures 28-31

Plants monoecious, without incrustations. Axes: 234-650 $\mu \mathrm{m}$ diam. Internodes: $1.1-1.8 \mathrm{~cm}$ long. Branchlets: monomorphic, 5-7, 2-3-furcated, 1.2-3.2 cm long. Primary rays: 5-7, 0.4-1.3 cm long. Secondary rays: $3-5$. Tertiary rays: $3-4$. Dactyls: 2-3, 2-3-celled, 156-5,590 $\mu \mathrm{m}$ long $\times 78-130 \mu \mathrm{m}$ diam., predominantly elongated, slender, occasionally abbreviated, penultimate cell cylindrical, end cell conical to acute. Heads: not formed, upper whorls occasionally compacted, no mucus. Gametangia: conjoined or sejoined at all furcations, occasionally absent at first. Oogonia: $1(-2)$ at a node, 350-463 $\mu \mathrm{m}$ long $\times$ 300-371 $\mu \mathrm{m}$ diam., convolutions 810 , coronula $38-52 \mu \mathrm{m}$ long $\times 51-65 \mu \mathrm{m}$ diam. Oospore: 299-319 $\mu \mathrm{m}$ long $\times 185-288 \mu \mathrm{m}$ diam., striae 6-7, fossa 43-63 $\mu \mathrm{m}$ across. Antheridia: 227-338 $\mu \mathrm{m}$ diam., octoscutate, triangular scute.

Representative specimens examined: BRAZIL, ParanÁ, Itaipu Reservoir: São Francisco Falso River, 11-II-2003, SM Thomaz \& TA Pagioro (UNOP1717); 11-II-2003, SM Thomaz \& TA Pagioro (UNOP1719); São Vicente River, 13-II-2003, SM Thomaz \& TA Pagioro (UNOP1779); 13-VIII-2002, SM Thomaz \& TA Pagioro (UNOP1797); 13-II-2003, SM Thomaz \& TA Pagioro (UNOP1814); Ocoí River, 19-II-2003, SM Thomaz \& TA Pagioro (UNOP1624); Passo Cuê River, 08-VIII-2002, SM Thomaz \& TA Pagioro (UNOP1646); 08-VIII-2002, SM Thomaz \& TA Pagioro (UNOP1647).

Geographic distribution in Brazil: Rio Grande do Sul (Astorino 1983, Prado 2003).

Comments: $N$. gracilis Smith $[=N$. gracilis (Smith) C.Agardh emend. R.D.Wood subsp. gracilis var. gracilis f. gracilis] is monoecious, has branchlets with two to three furcations, two- to three-celled dactyls, oospore with a finely granulated ornamentation pattern, with six to seven striae (Schubert \& Blindow 2004). The first record for Brazil was reported by Astorino (1983) in Rio Grande do Sul State. This is the first record of $N$. gracilis for the Itaipu Reservoir, and constitutes the first record of this species for Paraná State.

Some of the morphological structures differed in size as compared with those reported by Astorino (1983) and Prado (2003). The specimens from the São Vicente River (UNOP1814) had the largest and smallest oogonia, the specimens from the São Francisco Falso River (UNOP1717) had the smallest antheridia, and the specimens from the Ocoí River (UNOP1624) had the smallest oospores.

Nitella hyalina (DC.) C.Agardh, Systema Algarum, p.126. 1824.

Figures 32-34

Plants monoecious, delicate, with occasional carbonate incrustations. Axes: 234-390 $\mu \mathrm{m}$ diam. Internodes: $1 \mathrm{~cm}$ long, up to $9 \mathrm{X}$ the branchlet length. Branchlets: heteroclemous, 5-7, 1-2(-3)-furcated, 6-14 $\mathrm{mm}$ long. Primary rays: $5-7,2-5 \mathrm{~mm}$ long $\times 156-$ $260 \mu \mathrm{m}$ diam. Secondary rays: $2-5$. Tertiary rays: 3 . Dactyls: (2-)3(-4), 2-celled, 676-4,420 $\mu \mathrm{m}$ long $\times 78-$ $156 \mu \mathrm{m}$ diam. Accessory branchlets: in two whorls, 4-6, (0-)1-2-furcated, $1 / 2$ as long as primary rays. Gametangia: sejoined, with a short stalk, with mucus. Oogonia: solitary, 391-484 $\mu \mathrm{m}$ long $\times 319-381 \mu \mathrm{m}$ diam., convolutions 7-9, coronula 32-50 $\mu \mathrm{m}$ long, 50-65 $\mu \mathrm{m}$ diam. Oospore: 268-309 $\mu \mathrm{m}$ long $\times 185-278 \mu \mathrm{m}$ diam.; striae 6-7(-8), fossa $47-65 \mu \mathrm{m}$ across, membrane fibrous. Antheridia: 234-268 $\mu \mathrm{m}$ diam., octo-scutate, triangular scute.

Representative specimens examined: BRAZIL, PARANÁ, Itaipu Reservoir: Arroio Guaçu river, 11-VIII2002, SM Thomaz \& TA Pagioro (UNOP2904); São Francisco Verdadeiro River, 17-II-2003, SM Thomaz \& TA Pagioro (UNOP2197); São Vicente River, 13-II2003, SM Thomaz \& TA Pagioro (UNOP1783).

Geographic distribution in Brazil: Rio Grande do Sul (Prado \& Baptista 2005).

Comments: N. hyalina is monoecious and has two-celled dactyls and a solitary oogonia. The primary branchlets are long and have from two to four furcations, while the accessory short branchlets have from one to two furcations (Schubert \& Blindow 2004).

Some measurements of the specimens differed from those reported by Prado \& Baptista (2005). The upper morphometric limit for the dactyl length increased 

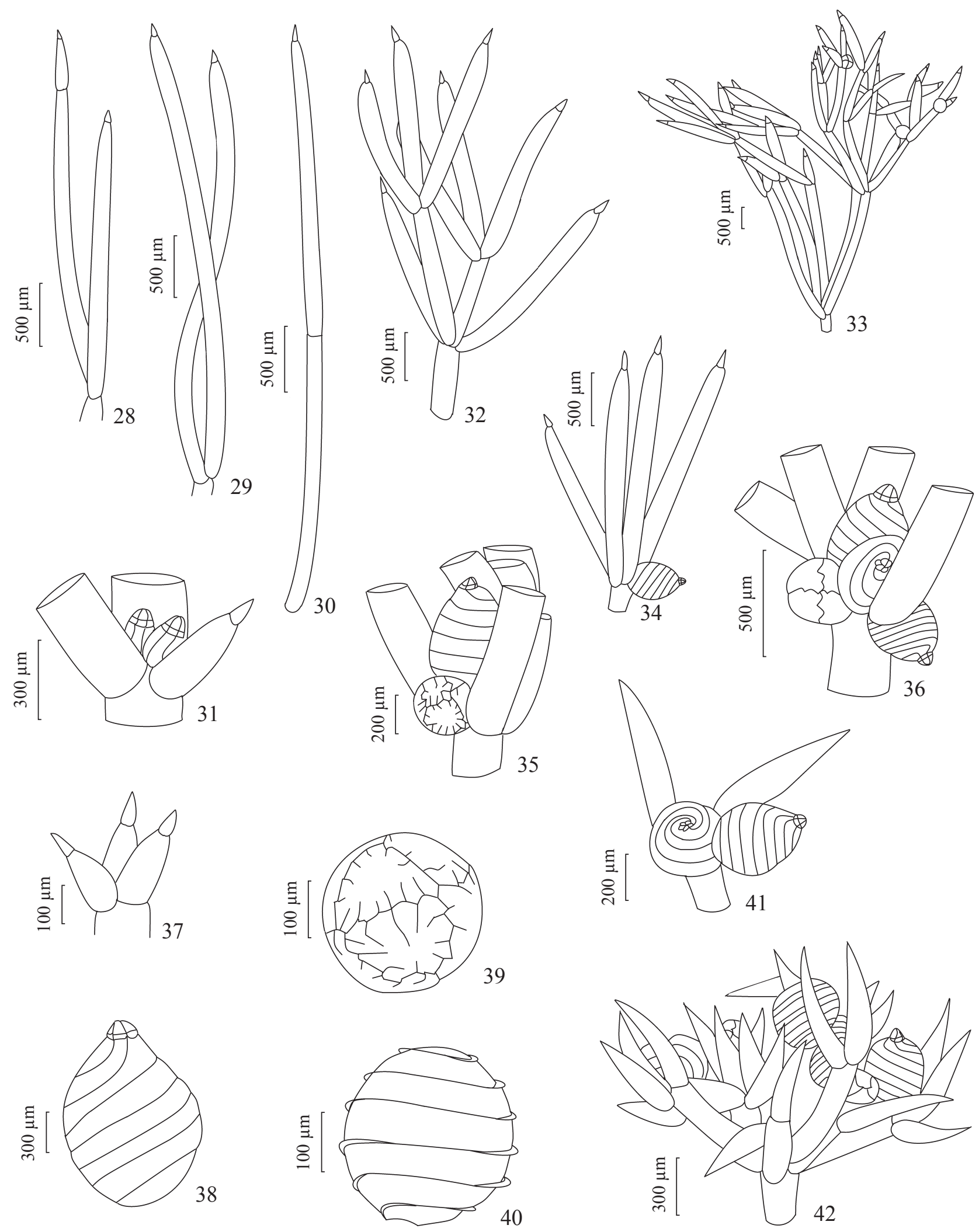

Figures 28-42. The genus Nitella from the Itaipu Reservoir. 28-31. Nitella gracilis. 28-30. Two and three-celled dactyls. 31. Fertile node. 32-34. N. hyaline. 32-33. Branchlets. 34. Fertile branchlet. 35-36. N. inversa, fertile nodes. 37-40. N. microcarpa. 37. Two-celled dactyls. 38. Oogonium. 39. Antheridium. 40. Oospore. 41-42. N. subglomerata. 41. Oogonium. 42. Fertile head. 
from $1350 \mu \mathrm{m}$ to $4420 \mu \mathrm{m}$, the lower limit of oogonia length decreased from $680 \mu \mathrm{m}$ to $484 \mu \mathrm{m}$, the lower limit of oospores decreased from $370 \mu \mathrm{m}$ to $286 \mu \mathrm{m}$, and the lower limits of antheridia from $330 \mu \mathrm{m}$ to $234 \mu \mathrm{m}$ diameter. The highest values for oogonia, coronula, oospores and dactyls were measured on specimens from sample UNOP1783, and the lowest values for antheridia, oospores, and dactyls were recorded on UNOP2197, both from the São Francisco River, while the specimens from the Arroio Guaçu River (UNOP2904) showed the lowest values for the oogonia, and the highest for the antheridia. This is the first record of $N$. hyalina in Paraná.

Nitella inversa Imahori, Japanese Charophyta. p. 125, pl.31, fig.44. 1954.

Figures 35-36

Plants monoecious. Stem: $13 \mathrm{~cm}$ tall; without incrustations. Axes: $442-728 \mu \mathrm{m}$ diam. Internodes: $1-3.6 \mathrm{~cm}$ long. Branchlets: monomorphic, 0.8-3.5 cm long, (2-)3-4-furcated. Primary rays: 6-8, 4-15 mm long. Secondary rays: 4-7. Tertiary rays: $3-4$. Quaternary rays: 2-3. Dactyls: 2-3, 2-3-celled, 208-1560 $\mu \mathrm{m}$ long $\times 78$ $156 \mu \mathrm{m}$ diam., predominantly abbreviated, penultimate cell cylindrical, end cell conical, apex acute. Heads: not formed. Gametangia: conjoined and sejoined at all furcations, occasionally with a short stalk. Oogonia: 1-3 per node, terminal, 443-567 $\mu \mathrm{m}$ long $\times 319-402 \mu \mathrm{m}$ diam., convolutions $8-9$, coronula 42.5-82 $\mu \mathrm{m}$ long $\times 60$ $93 \mu \mathrm{m}$ diam. Oospore: $278-330 \mu \mathrm{m}$ long $\times 337-288 \mu \mathrm{m}$ diam., striae $6-7$, fossa $50-55 \mu \mathrm{m}$ across, membrane reticulate. Antheridia: terminal or lateral, 237-329 $\mu \mathrm{m}$ diam., octo-scutate, triangular scute.

Representative specimens examined: BRAZIL, PARANÁ, Itaipu Reservoir: São Vicente River, 13-II-2003, SM Thomaz \& TA Pagioro (UNOP1786); Ocoí River, 15-VIII-2002, SM Thomaz \& TA Pagioro (UNOP1616); 14-VIII-2002, SM Thomaz \& TA Pagioro (UNOP1629); Passo Cuê River, 08-VIII-2002, SM Thomaz \& TA Pagioro (UNOP1657).

Geographic distribution in Brazil: [as Nitella furcata f. inversa R.D.Wood] São Paulo (Picelli-Vicentim et al. 2004) and Rio Grande do Sul (Prado 2003).

Comments: This is the first record of $N$. inversa Imahori [sin. Nitella furcata (Roxburgh ex Bruzelius) C.Agardh emend. R.D.Wood subsp. furcata var. sieberi (A.Braun) R.D.Wood f. inversa (Imahori) R.D.Wood] in the Itaipu Reservoir, and is also the first report of this species in Paraná State. Nitella inversa has terminal oogonia, terminal or lateral antheridia (PicelliVicentim et al. 2004), two- and three-celled dactyls, and ornamentation of the oospore membrane varying from papillate to reticular (Sakayama 2008).

Compared to the specimens observed by Prado (2003), the material analyzed here showed differences in the morphometric limits of the oospores (from $320 \mu \mathrm{m}$ to $278 \mu \mathrm{m}$ long) and antheridia (from $310 \mu \mathrm{m}$ to $329 \mu \mathrm{m}$ diameter). The highest and the lowest morphometric values for the oogonia and lowest value of the antheridia were recorded in the sample from the São Vicente River (UNOP1786). The specimens from the Ocoí River showed the smallest measurements for oospores (UNOP1616 and UNOP1629) and the largest measurements for antheridia (UNOP1629).

Nitella microcarpa A.Braun. Monatsbericht der deutschen Akademie der Wissenschaften zu Berlin 357. 1858.

Figures 37-40

Plants monoecious. Stem: $10 \mathrm{~cm}$ tall, without incrustations. Axes: 390-702 $\mu \mathrm{m}$ diam. Internodes: 1.6-4.2 cm long. Branchlets: monomorphic, 7-8, 2-3 furcated, $1.2-2.7 \mathrm{~cm}$ long. Primary rays: $7-8,0.4-1 \mathrm{~cm}$ long. Secondary rays: $4-5,1$ of which is occasionally central and reduced. Tertiary rays: $3-4$. Quaternary rays: 2-3. Dactyls: 2-3, 2-3-celled, 94-2,392(-3,510) $\mu \mathrm{m}$ long $\times$ 41-182 $\mu \mathrm{m}$ diam., predominantly abbreviated, penultimate cell cylindrical, end cell conical, acute apex. Heads: not formed. Gametangia: conjoined at all furcations. Oogonia: 2-3 at a node, 412-525 $\mu \mathrm{m}$ long $\times$ 268-443 $\mu \mathrm{m}$ diam., convolutions 7-8, coronula 45-65 $\mu \mathrm{m}$ long $\times 62-82 \mu \mathrm{m}$ diam. Oospore: $288-309 \mu \mathrm{m}$ long $\times$ 247-299 $\mu \mathrm{m}$ diam., striae 5-6, fossa 50-57 $\mu \mathrm{m}$ across, membrane reticulate. Antheridia: 227-494 $\mu \mathrm{m}$ diam., octo-scutate, triangular scute.

Representative specimens examined: BRAZIL, PARANÁ, Itaipu Reservoir: São João River, 12-VIII-2002, SM Thomaz \& TA Pagioro (UNOP1739); 12-VIII-2002, SM Thomaz \& TA Pagioro (UNOP1772); Ocoí River, 14-VIII-2002, SM Thomaz \& TA Pagioro (UNOP1611); 24-VIII-2002, SM Thomaz \& TA Pagioro (UNOP2081); 14-VIII-2002, SM Thomaz \& TA Pagioro (UNOP2917); Pinto River, 09-VIII-2002, SM Thomaz \& TA Pagioro (UNOP2062); Passo Cuê River, 09-VIII-2002, SM Thomaz \& TA Pagioro (UNOP1650).

Geographic distribution in Brazil: [as Nitella furcata f. microcarpa (A.Braun) R.D.Wood] Mato Grosso do Sul (Bueno \& Bicudo 1997) and São Paulo State (Necchi Júnior et al. 2000, Picelli-Vicentim et al. 2004).

Comments: Braun (1858) first reported the occurrence of this species in Brazil. Nitella microcarpa has two to three oogonia at a single node, predominantly abbreviated 
dactyls with two and three cells, and the axis up to $1 \mathrm{~mm}$ in diameter. The material analyzed here increased the upper limit for dactyl length from $2600 \mu \mathrm{m}$ (Picelli-Vicentim et al. 2004) to $3510 \mu \mathrm{m}$, oospore morphometric limits increased from $292 \mu \mathrm{m}$ long $\times 260 \mu \mathrm{m}$ diameter (Bueno $\&$ Bicudo 1997) to $309 \mu \mathrm{m}$ long $\times 299 \mu \mathrm{m}$ diam., and the morphometric limits of the antheridia increased from $290 \mu \mathrm{m}$ (Bueno \& Bicudo 1997) to $300 \mu \mathrm{m}$ diameter.

Nitella subglomerata A.Braun 1858. Monatsbericht der deutschen Akademie der Wissenschaften zu Berlin 356. 1858.

Figures 41-42

Plants monoecious, without incrustations. Axes: 468-832 $\mu \mathrm{m}$ diam. Internodes: $1.3-2 \mathrm{~cm}$ long. Branchlets: dimorphic. Sterile branchlets: 6-10, 0.9-2.3 cm long, 1 -furcated, primary rays $6-10,0.8-1.7 \mathrm{~cm}$ long, dactyls 2-5, 1-celled, acuminate apex, 1300-9000 $\mu \mathrm{m}$ long $\times$ 117-312 $\mu \mathrm{m}$ diam. Fertile branchlets: reduced, forming heads, 7-10, 453-3952 $\mu \mathrm{m}$ long $\times$ 72-104 $\mu \mathrm{m}$ diam., 1-furcated, dactyls 3-4, elongated, curved, acuminate apex, 1-celled, 463-858 $\mu \mathrm{m}$ long $\times$ 72-104 $\mu \mathrm{m}$ diam. Heads: stipitate, numerous, 1-3 per whorl, semi-spherical to conical, lax, occasionally forming whorls with axillary ramifications, 0.1-2 mm diam. Gametangia: conjoined or sejoined. Oogonia: $1-3$ at a node, 309-433 $\mu \mathrm{m}$ long $\times$ 268-309 $\mu \mathrm{m}$ diam., convolutions 8-9, coronula 28-42 $\mu \mathrm{m}$ long $\times$ 41-50 $\mu \mathrm{m}$ diam. Oospore: $206-278 \mu \mathrm{m}$ long $\times$ 237-546 $\mu \mathrm{m}$ diam., striae 6-7, fossa $42-45 \mu \mathrm{m}$ across, membrane granulate. Antheridia: 237-278 $\mu \mathrm{m}$ diam., octo-scutate, triangular scute.

Representative specimens examined: BRAZIL, PARANÁ, Itaipu Reservoir: São Francisco Falso River, 25-IV-2002, SM Thomaz \& TA Pagioro (UNOP1354); São João River, 20-II-2003, SM Thomaz \& TA Pagioro (UNOP2916); 20-II-2003, SM Thomaz \& TA Pagioro (UNOP2176); Ocoí River, 28-I-2002, SM Thomaz \& TA Pagioro (UNOP1312); Pinto River, 23-I-2002, SM Thomaz \& TA Pagioro (UNOP2897).

Geographic distribution in Brazil: in the states of Mato Grosso (Braun \& Nordstedt 1883, Bicudo \& Yamaoka 1978), Mato Grosso do Sul (Bicudo \& Yamaoka 1978, Bueno \& Bicudo 1997), Minas Gerais (Braun \& Nordstedt 1883, Bicudo 1969, Bicudo \& Yamaoka 1978), Paraná (Bicudo \& Yamaoka 1978), Rio Grande do Sul (Astorino 1983, Prado 2003), and São Paulo (Braun \& Nordstedt 1883, Wood \& Imahori 1965, Bicudo \& Yamaoka 1978, Picelli-Vicentim \& Bicudo 1993, Necchi Junior et al. 2000, Vieira Júnior et al. 2002, PicelliVicentim et al. 2004).
Comments: N. subglomerata is cosmopolitan and widely recorded in Brazil. The material analyzed here was similar to that reported by Bicudo \& Yamaoka (1978), Astorino (1983), Bueno \& Bicudo (1997), Picelli-Vicentim \& Bicudo (1993), and Picelli-Vicentim et al. (2004). The species has fertile branchlets reduced into heads, one-celled dactyls, and one-furcated dimorphic branchlets. The presence of fertile heads distinguishes $N$. subglomerata from $N$. acuminata, and a monoecious condition separates it from the dioecious species N. blankinshipii T.F.Allen. Specimen UNOP2176 showed the lowest and highest morphometric values for antheridia, and UNOP2916 the lowest values for oogonia and oospores.

\section{General comments}

Information regarding the occurrence of the genera Chara and Nitella in Paraná State is still relatively scarce. Chara guairensis was described by Bicudo (1972, 1974) for the Guaíra region, N. subglomerata was recorded for the mid-western region of the state (Branco et al. 2009), the genus Nitella was recorded for the mid-southern region (Krupek et al. 2008), and $N$. furcata recorded for the Upper Paraná river floodplain (Thomaz et al. 2009). Species recorded for the Itaipu Reservoir include C. braunii, C. guairensis, Chara sp., Nitella sp., N. acuminata, N. furcata, N. furcata subsp. mucronata, and N. subglomerata (Thomaz et al. 1999, 2003, Mormul et al. 2010). Recently, Bueno et al. (2011) contributed with records of $C$. diaphana and $C$. kenoyeri as new citations for the reservoir.

Nine species of Nitella were recorded. The Ocoí River contained the highest species richness (seven), followed by the São João and São Vicente rivers with six species each. These areas, located in the lacustrine zone of the reservoir, are mesotrophic to oligotrophic (Bini et al. 1999) and favorable environments for the development of submerged algae. The Arroio Guaçu and São Francisco Verdadeiro rivers, located in the transition and fluvial zones of the reservoir respectively, contained one and two species respectively. These regions have eutrophic characteristics (Bini et al. 1999), with frequent phytoplankton blooms that make the environment less favorable to the development of submerged species such as the Characeae. The differences in Characeae richness seen among the different tributaries of the fluvial, transition, and lacustrine zones are in accordance with the results of Pagioro \& Thomaz (2002) who found a gradient of sedimentation rates in the reservoir, where water 
transparency gradually increased from the fluvial zone to the lacustrine zone, allowing greater colonization by submerged plants in the clearer water.

The species Chara hydropitys, C. rusbyana, Nitella axillaris, $N$. glaziovii, $N$. gracilis, $N$. hyalina, $N$. inversa, and $N$. microcarpa are new records for the Itaipu Reservoir and Paraná State. We believe that the differences in richness between the present study and those reported by Thomaz et al. (1999, 2003), Mormul et al. (2010), and Bueno et al. (2011) were due to different criteria for sampling and for identification, and to the taxonomic resolutions used in the different studies.

The morphometric differences seen between the specimens analyzed and those previously described in the studies of Bicudo (1972, 1974), Astorino (1983), Bueno et al. (1996), Prado (2003), Vieira Júnior et al. (2003, 2003), Picelli-Vicentim et al. (2004), and Bueno et al. $(2009,2011)$ could represent variation due to phenotypic plasticity, corresponding to the different environmental conditions to which these plants were subjected (Asaeda et al. 2007, Blindow \& Schütte 2007), although further investigations are necessary to confirm this hypothesis.

Acknowledgments - The authors thank the anonymous reviewers and the editors for careful reviewing this manuscript; the curators of the herbaria mention above for specimen loans and for access to their collections; the Itaipu Technological Park Foundation/Foz do Iguaçu/PR (Fundação Parque Tecnológico Itaipu - FPTI) for the grant; and Sidinei Magela Thomaz (UEM/Nupelia) for supplying biological materials and providing us with assistance.

\section{REFERENCES}

Asaeda T, Rajapakse L, Sanderson B. 2007. Morphological and reproductive acclimations to growth of two charophyte species in shallow and deep water. Aquatic Botany 86:393-401.

Astorino HAB. 1983. Charophyceae do Estado do Rio Grande do Sul: uma contribuição do seu inventário. Tese de mestrado, Universidade Estadual Paulista, Rio Claro.

Bicudo RMT. 1969. Brazilian Characeae of the herbarium of the Instituto de Botânica. Nova Hedwigia 17:1-17.

Bicudo RMT. 1974. O gênero Chara (Charophyceae) no Brasil, 1: Subseção Willdenowia RD Wood. Rickia 6:127-189.

Bicudo RMT. 1979. O gênero Chara (Charophyceae) no Brasil, 3: Seção Charopsis (Kützing emend. Rupr., Leonh.) RD Wood. Rickia 8:17-26.

Bicudo RMT, Yamaoka DM. 1978. O gênero Nitella (Charophyceae) no Brasil, 1: Subgênero Nitella. Acta Biologica Paranaense 7:77-98.
Bini LM, Thomaz SM, Murphy KJ, Camargo AFM. 1999. Aquatic macrophyte distribution in relation to water and sediment conditions in the Itaipu Reservoir, Brazil. Hydrobiologia 415:147-154.

Blindow I, Schütte M. 2007. Elongation and mat formation of Chara aspera under different light and salinity conditions. Hydrobiologia 584:69-76.

Branco CCZ, Peres CK, Krupek RA, Bertusso FR. 2009. Macroalgas de ambientes lóticos da região centro-oeste do Paraná, região sul do Brasil. Biota Neotropica 9: 227-236.

Braun A. 1858. Characeen aus Columbien, Guyana und Mittelamerika. Monatsbericht der Königlichen Akademie der Wissenschaften zu Berlin vom Juni 1858:349-368.

Braun A, Nordstedt CFO. 1883. Fragmente einer Monographie der Characeen: nach den hinterlassenen Manuscripten. A. Braun's herausgegeben von Dr. Otto Nordstedt. Abhandlungen der Königinen Akademie der Wissenschaftlichen Berlim 1882:1-211.

Bueno NC, Bicudo CEM. 1997. Characeae (Charophyceae) do Pantanal de Mato Grosso do Sul, Brasil: Nitella. Hoehnea 24:29-55.

Bueno NC, Bicudo CEM, Picelli-Vicentim MM, Ishii II. 1996. Characeae (Charophyceae) do Pantanal de Mato Grosso do Sul, Brasil: Chara. Hoehnea 24:29-55.

Bueno NC, Bicudo CEM, Biolo S, Meurer T. 2009. Levantamento taxonômico das Characeae (Chlorophyta) de Mato Grosso e Mato Grosso do Sul, Brasil: Chara. Revista Brasileira de Botânica 32:735-750.

Bueno NC, Prado JF, Meurer T, Bicudo CEM. 2011. New records of Chara (Chlorophyta, Characeae) for subtropical Southern Brazil. Systematic Botany 36: 523-541.

Casanova MT. 2005. An overview of Chara L. in Australia (Characeae, Chlorophyta). Australian Systematic Botany 18:25-39.

Coops H. 2002. Ecology of charophytes: an introduction. Aquatic Botany 72:205-208.

Groves H, Groves J. 1911. Characeae. In Symbolae Antillanae: seu Fundamenta Florae Indiae Occidentalis 7 (I Urban, ed.). Fratres Borntraeger, Berlin.

Horn af Rantzein H. 1949. Charophyta reported from Latin America. Arkiv för Botanik 1:355-411.

Howe MA. 1929. Two new species of Chara from tropical Latin America. Field Museum of Natural History 4: 159-161.

Karol KG, Mccourt RM, Cimino MT, Delwiche CF. 2001. The closest living relatives of land plants. Science 294:2351.

Krause W. 1997. Charales (Charophyceae). Sußwasserflora von Mitteleuropa. v.18. (H Ettl, G Gärtner, H Heyning, D Molenhauser, eds.). Gustav Fischer Verlag, Sttutgart.

Krupek RA, Branco CCZ, Peres CK. 2008. Levantamento florístico das comunidades de macroalgas da bacia do Rio das Pedras, região centro-sul do Estado do Paraná, Sul do Brasil. Hoehnea 35:189-208. 
Kufel L, Kufel I. 2002. Chara beds acting as nutrient sinks in shallow lakes - a review. Aquatic Botany 72:249-260.

Mandal DK, Ray S, Mukherjee A. 1995. Scanning electron microscopic study of compound oospore wall ornamentations in some taxa under Nitella furcata complex (Charophyta) from India. Phytomorphology 45:39-45.

Meurer T, Biolo S, Bortolini JC, Bueno NC. 2008. Characeae (Chlorophyta) do Reservatório de Itaipu: Chara braunii Gmelin. Revista Brasileira de Biociências 6:3-4.

Moore JA. 1986. Charophytes of Great Britain and Ireland. Botanical Society of the British Isles, London.

Mormul RP, Ferreira FA, Michelan TS, Carvalho P, Silveira MJ, Thomaz SM. 2010. Aquatic macrophytes in the large, sub-tropical Itaipu Reservoir, Brazil. Revista de Biología Tropical 58:1437-1452.

Necchi Júnior O, Pascoaloto D, Branco LHZ. 1994. Distribution of macroalgae in tropical river basin from southeastern Brazil. Archiv für Hydrobiologie 129: 459-471.

Necchi Júnior O, Pascoaloto D, Branco LHZ. 1997. Stream macroalgal flora from the northwest region of São Paulo state, southeastern Brazil. Algological Studies 84: 91-112.

Necchi Júnior O, Branco CCZ, Branco LHZ. 2000. Distribution of stream macroalgae in São Paulo State, southeastern Brazil. Algological Studies 97:43-57.

Pagioro TA, Thomaz SM. 2002. Longitudinal patterns of sedimentation in a deep, monomictic subtropical reservoir (Itaipu, Brazil-Paraguay). Archiv für Hydrobiologie 154:515-528.

Picelli-Vicentim MM, Bicudo CEM. 1993. Criptógamos do Parque Estadual das Fontes do Ipiranga, São Paulo, SP. Algas, 4: Charophyceae. Hoehnea 20:9-22.

Picelli-Vicentim MM, Bicudo CEM, Bueno NC. 2004. Flora ficológica do Estado de São Paulo, 5: Charophyceae. São Paulo: RiMa Editora.

Prado JF. 2003. Characeae do Rio Grande do Sul, Brasil. Tese de doutorado, Universidade Federal do Rio Grande do Sul, Porto Alegre.

Prado JF, Baptista LRM. 2005. Novos registros de Characeae (Chlorophyta) para o Brasil. Iheringia: série Botânica 60:259-268.

Proctor VW, Griffin III DG, Hotchkiss AT. 1971. A sinopsis of the genus Chara, series Gymnobasalia (subsection Willdenowia RD Wood). American Journal of Botany 58:894-901.

Rodrigo MA, Rojo C, Alvarez-Cobelas M, Cirujano S. 2007. Chara hispida beds as a sink of nitrogen: evidence from growth, nitrogen uptake and decomposition. Aquatic Botany 87:7-14.
Sakayama H, Nozaki H, Kasaki H, Hara Y. 2002. Taxonomic re-examination of Nitella (Charales, Charophyceae) from Japan, based on microscopical studies of oospore wall ornamentation and $r b c L$ gene sequences. Phycologia 41:397-408.

Sakayama H. 2008. Taxonomy of Nitella (Charales, Charophyceae) based on comparative morphology of oospore and multiple DNA marker phylogeny using cultured material. Phycological Research 56:202-215.

Schubert H, Blindow I. 2004. Charophyte of the Baltic Sea. A.R.G. Gäntner Verlag Kommanditgesellschaft, Ruggell.

Schwarz A-M, Winton M, Hawes I. 2002. Species-specific depth zonation in New Zealand charophytes as a function of light availability. Aquatic Botany 72:209-217.

Thomaz SM, Bini LM, Souza MC, Kita KK, Camargo AFM. 1999. Aquatic macrophytes of Itaipu Reservoir, Brazil: survey of species and ecological considerations. Brazilian Archives of Biology and Technology 42: 15-22.

Thomaz SM, Souza DC, Bini LM. 2003. Species richness and beta diversity of aquatic macrophytes in a large sub-tropical reservoir (Itaipu Reservoir, Brazil): the influence of limnology and morphometry. Hydrobiologia 505:119-128.

Thomaz SM, Carvalho P, Mormul RP, Ferreira FA, Silveira MJ, Michelan TS. 2009. Temporal trends and effects of diversity on occurrence of exotic macrophytes in a large reservoir. Acta Oecologica 35:614-620.

Van Donk E, Van de Bund E. 2002. Impact of submerged macrophytes including charophytes on phyto- and zooplankton communities: allelopathy versus other mechanisms. Aquatic Botany 72:261-274.

Vieira Júnior J, Necchi Júnior O. 2002. Microhabitat and plant structure of Characeae (Chlorophyta) populations in streams from southeastern Brazil. Cryptogamie Algologie 23:51-63.

Vieira Júnior J, Necchi Júnior O, Branco CCZ, Branco LHZ. 2002. Characeae (Chlorophyta) em ecossistemas lóticos do Estado de São Paulo, Brasil: gênero Nitella. Hoehnea 29:249-266.

Vieira Júnior J, Necchi Júnior O, Branco CCZ, Branco LHZ. 2003. Characeae (Chlorophyta) em ecossistemas lóticos do Estado de São Paulo, Brasil: gênero Chara e distribuição ecológica. Hoehnea 30:53-70.

Wood RD, Imahori K. 1964. A revision of the Characeae, 2: Iconograph of the Characeae. J. Cramer, Weinhen.

Wood RD, Imahori K. 1965. A revision of the Characeae, 1: monograph of the Characeae. J. Cramer, Weinhen.

Zaneveld JS. 1940. The Charophyta of Malaysia and adjacent countries. Blumea 4:1-224. 